\title{
MICROWAVE-DRIVEN MULTIFUNCTIONAL CAPABILITY OF MEMBRANE STRUCTURES
}

\author{
Sang H. Choi*, Sang-Hyon Chu*, Kyo D. Song $\dagger$, and Glen C. King* \\ *NASA Langley Research Center, Hampton, Virginia 23681-2199 \\ $\uparrow$ Norfolk State University, Norfolk, Virginia 23504
}

\begin{abstract}
A large, ultra lightweight space structure, such as solar sails and Gossamer spacecrafts, requires a distributed power source to alleviate wire networks, unlike the localized on-board power infrastructures typically found in most small spacecrafts. The concept of microwave-driven multifunctional capability for membrane structures is envisioned as the best option to alleviate the complexity associated with hard-wired control circuitry and on-board power infrastructures. A rectenna array based on a patch configuration for high voltage output was developed to drive membrane actuators, sensors, probes, or other devices. Networked patch rectenna array receives and converts microwave power into a DC power for an array of smart actuators. To use microwave power effectively, the concept of a power allocation and distribution (PAD) circuit is adopted for networking a rectenna/actuator patch array. The use of patch rectennas adds a significant amount of rigidity to membrane flexibility and they are relatively heavy. A dipole rectenna array (DRA) appears to be ideal for thin-film membrane structures, since DRA is flexible and light. Preliminary design and fabrication of PAD circuitry that consists of a few nodal elements were made for laboratory testing. The networked actuators were tested to correlate the network coupling effect, power allocation and distribution, and response time.
\end{abstract}

\section{INTRODUCTION}

A microwave-driven smart membrane system provides multifunctional capabilities to ultra-light weight structures, such as the shape control of inflatable membrane space antennas, smart-wing concept of future aircrafts, unmanned autonomous aircraft vehicles (UAV), and insect-like flying objects development. ${ }^{1,2}$ The microwave-driven smart membrane actuator system is based on a wireless power transmission to actuators without a complex wiring and gate switching. This can reduce the total weight and fabrication cost of the system. Smart materials, such as piezoelectric and electroactive polymers (EAP), undergo shape changes along with applied electric field in a predictable way ${ }^{3,4}$ and are used for actuator applications. Recently, a smart material actuator was utilized for a surface compensation of inflatable reflector antenna. ${ }^{5}$ The surface compensation technique is extremely crucial to the success of NASA's future missions ${ }^{6-9}$ including the Gossamer spacecrafts, Next Generation Space Telescope (NGST), space relay antennas, aircraft morphing, and insect-like flying objects.

For a system with numerous nodal points that require power feed and control, the hard wiring may not be a suitable solution due to the network complexity, the weight increase attributed to a wired network, the complex gate switching of power and control networks, and the interdependency of power and control routines. However, these shortcomings of wire-networked system may be alleviated by adopting a wireless power transmission with beam steering and tailoring using microwave properties such as power, frequency, and polarization.

Since the rectenna was first introduced by W. C. Brown in the $1960^{\prime} \mathrm{s},{ }^{10}$ it has been used for various applications such as a microwave-powered helicopter and model airplanes, ${ }^{11}$ and later proposed for the Solar Power Satellite (SPS) ${ }^{12}$ that converts solar energy to $\mathrm{RF}$ and beams down to large $2.45-\mathrm{GHz}$ rectennas on Earth, the 4.5-meter wingspan airplane that was powered only by microwave energy, ${ }^{13}$ and a microwave-powered balloon with an electronically steerable phased array. ${ }^{14}$ Most smart materials, such as piezoelectric or electroactive, require electrical power in a range of 200 - 300 volts to activate appreciable shape changes. A $3 \times 3$ array of patch rectennas was developed in our earlier efforts for a multilayered piezoelectric actuator. It was able to generate an output voltage of 70 volts. ${ }^{1,2}$ Although the result was not impressive, it laid out the direction for next generation rectenna devices. Recently, a lightweight patch rectenna was developed at NASA's Jet Propulsion Laboratory. ${ }^{15}$ The improved rectenna is a square planar array of identical unit cells that have a receiving antenna, filter and rectifier circuitry in its planar structure. Microwave coupling between each copper antenna patch and its underlying filter and rectifier circuit occurs in two orthogonal slots in the copper ground plane.

The power output harvested from patch rectennas is determined by the power flux density, frequency, incident angle of incident microwave, and rectifying circuit performance. Devices for aerospace applications are typically designed for high power density (volumetric or mass-based) and low power 


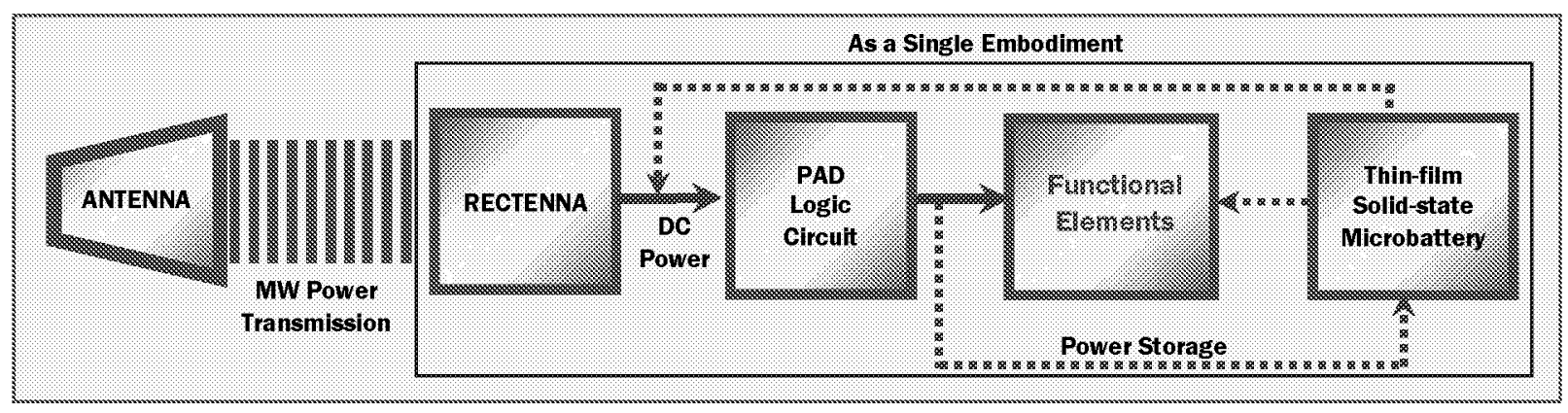

Fig. 1. Microwave driven multifunctional capabilities for smart membrane structures.

requirement. In addition to being lightweight, thin and flexible, microwave-driven smart membrane actuators must meet these power requirements. Devices operating at a low power level should intelligently manage the power. Normally, high energy and high frequency microwave sources are bulky and heavy, and thus inappropriate for space applications. The issues related to low power density of microwave can be easily addressed by adopting the concept of power allocation and distribution (PAD).

Also, the type of power for space-based or airborne structures is mode-dependent, either continuous or pulse. The imbedded nature of power harvesting, distribution, storage, and control for shape changes, data gathering, and probing within a single embodiment, as shown in Fig. 1, impose very stringent design criteria as thin-film for flexible structures, micro or nano sizes, ultra lightweight elements, less complexity, and enhancement. The power mode for a selected functional element is modulated for either high voltage or current according to the need. For the purpose of the shape control, the rectenna needs to

$$
\text { Resistance }-100 \mathrm{kR}
$$

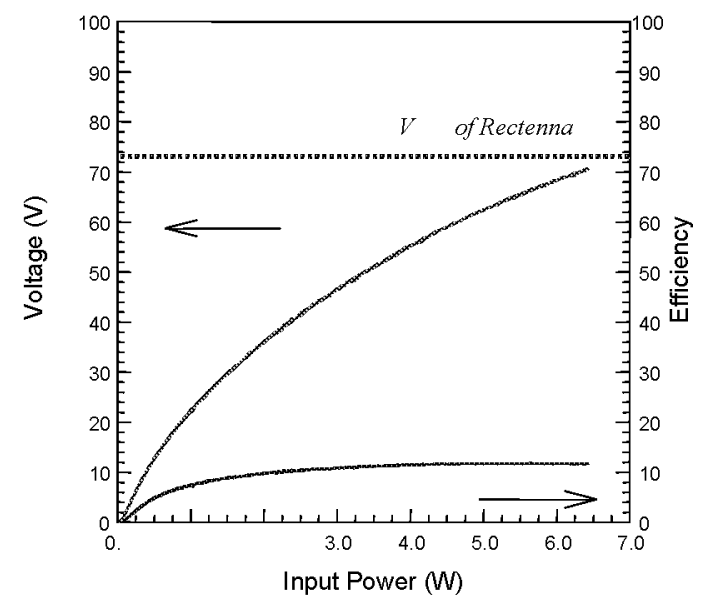

provide either high voltage/low current for piezoelectric and electro-active materials, or low voltage/high current for magnetostrictive materials. Other functional elements do not need power mode and modulation as extreme as required for shape control devices.

\section{EXPERIMENTAL RESULTS}

In this study, the multilayer piezoelectric actuators were chosen since they exhibit high longitudinal displacement, fast response, low driving voltage, and high energy conversion efficiency. ${ }^{16} \mathrm{~A}$ JPL $3 \times 3$ patch rectenna was used and characterized in various conditions, such as transmitted power, frequency, beam incident angle, and beam polarization. A $100 \mathrm{k} \Omega$ loaded rectenna was tested to apply a high voltage to the actuator and maximize its performance. In this case, it was possible to obtain up to $73 \mathrm{~V} \mathrm{DC}$, whereas the efficiency was only $12 \%$ as shown in Fig. 2 . The effect

$$
\text { Resistance }-5.4 \mathrm{~km}
$$

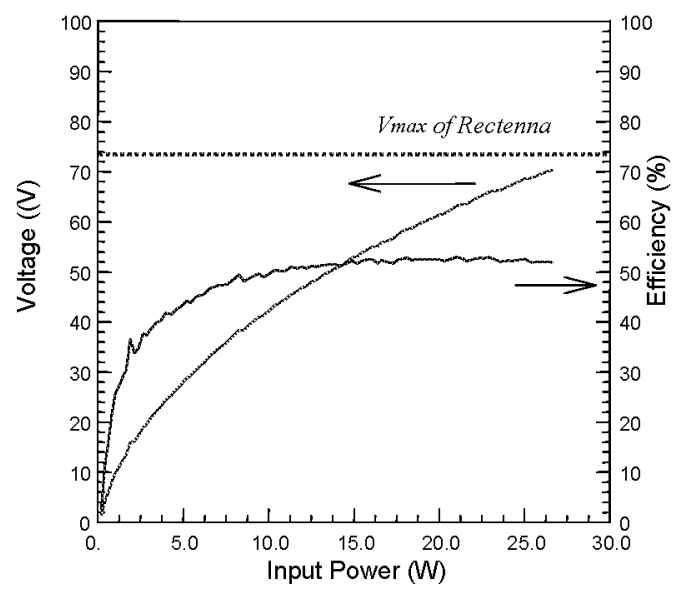

Fig. 2. Transmitted power effect on voltage output and efficiency obtained by $3 \times 3$ patch rectenna with 100,000 and $5,400 \mathrm{Ohms}$ at a distance of $38 \mathrm{~cm}$ from feed horn at constant frequency of $8.5 \mathrm{GHz}$. 


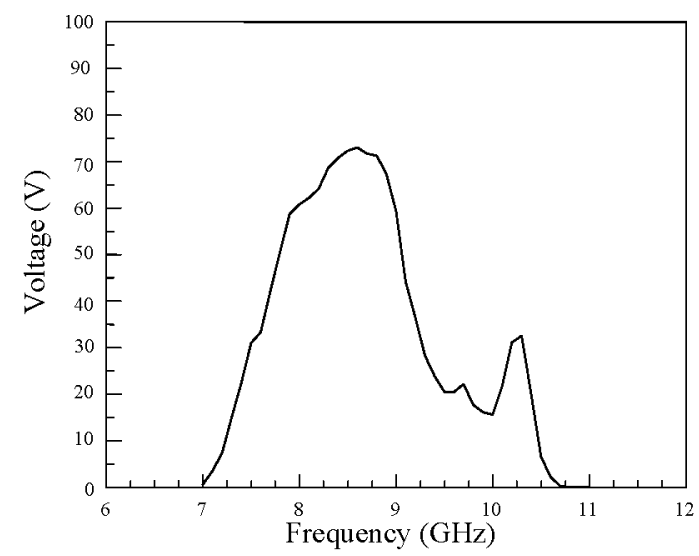

Fig. 3. Frequency effect on voltage output generated by $3 \times 3$ patch rectenna at $63.5 \mathrm{~cm}$ from feed horn with constant transmitted power of $1.1 \mathrm{~W}$.

of frequency on rectenna performance was tested at a constant power, $1.1 \mathrm{~W}$, when the patch rectenna was placed at a distance of $100 \mathrm{~cm}$ away from the feed horn. In Figs. 2 and 3, it is shown that the maximum voltage of $73 \mathrm{~V}$ DC was produced at $8.6 \mathrm{GHz}$. Fig. 4 illustrates that the displacement of multilayer piezoelectric actuator powered by the rectenna's DC voltage output up to about $70 \mathrm{~V}$. The nearly linear relationship between the displacement and the applied voltage signifies nearly constant $d_{33}(=0.157[\mu \mathrm{m} / \mathrm{V}])$ over a whole test range. At $50 \mathrm{~V}$, the displacement was measured to be around $8.0 \mu \mathrm{m}$, which is much larger than the $5.6 \mu \mathrm{m}$ that was obtained with the same level of $\mathrm{AC}$ voltage at $0.5 \mathrm{~Hz}$. At this frequency, however, dielectric loss due to the high frequency is expected to be negligible. Thus, if RMS value of AC voltage is considered instead of the peak-to-peak value, we obtain good agreement between the $\mathrm{AC}$ source at $0.5 \mathrm{~Hz}$ and the rectenna power, as shown in Fig. 4.

To extend our research, the $6 \times 6$ patch rectennas shown

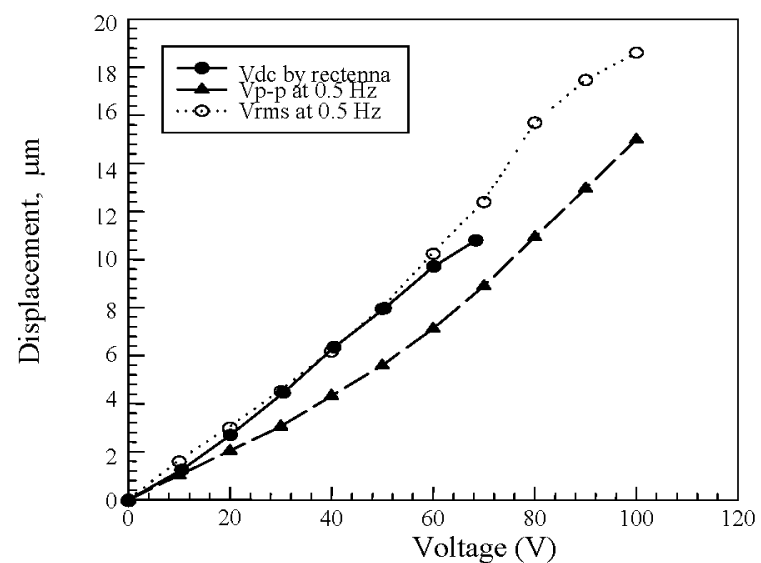

Fig. 4. Displacement of multilayer piezoelectric actuator activated by various power sources.

in Fig. 5 were built for high voltage output. The voltage output of patch rectennas is determined by the number of embedded Schottky barrier diodes on a rectifying circuit. Each patch consists of two of Schottky barrier diodes embedded in a serial mode that hold off the total of 15 volts. Thus, a $6 \times 6$ patch rectenna is, theoretically, able to generate the output voltage up to 540 volts. The actual voltage output is lower than the theoretical value and determined by the electrical properties of substrate materials on which a rectifying circuit is built. The performance of a $6 \times 6$ patch rectenna is shown in Figs 6 and 7. The power outputs $\left(\approx V^{2} / R_{L}\right)$ from a $6 \times 6$ patch rectenna module with constant load resistance $\left(\mathrm{R}_{\mathrm{L}}\right)$ were measured at a farfield along the distance from the feed horn and plotted in terms of voltage output versus distance as shown in Fig. 6. The frequency-dependent voltage outputs were also measured at the distance of $100 \mathrm{~cm}$ away from a feed horn as shown in Fig. 7. The peak voltage output was 230 volts at $8 \mathrm{GHz}$ with 20 watts of microwave
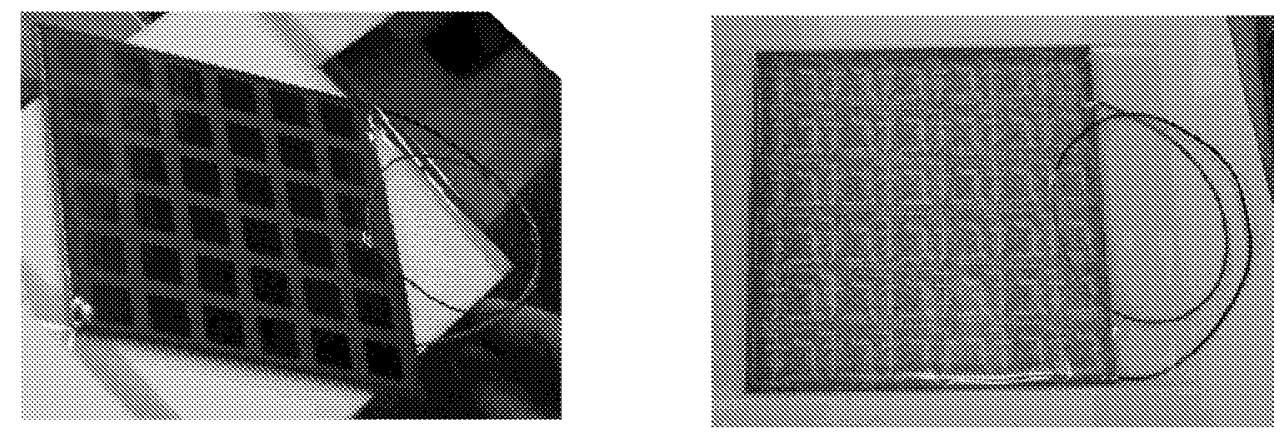

Fig. 5. A $6 \times 6$ patch rectenna array 


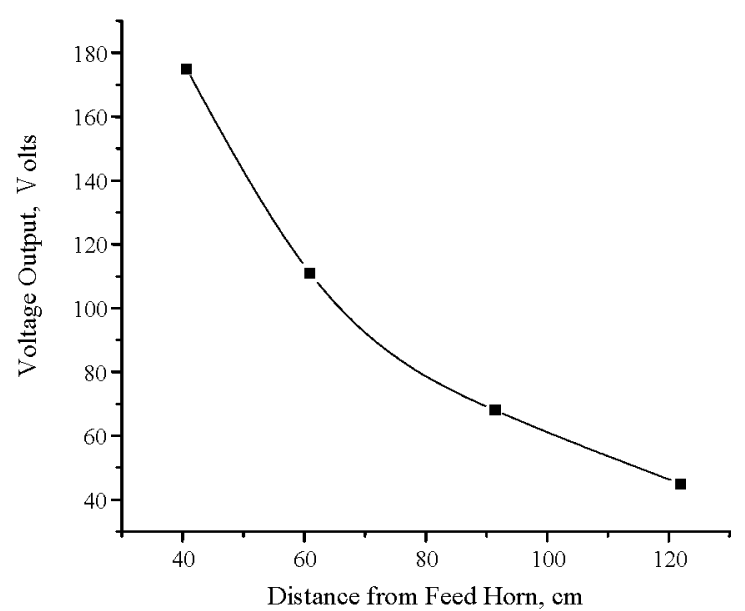

Fig. 6. Voltage outputs from a $6 \times 6$ patch rectenna array with distance from feed horn

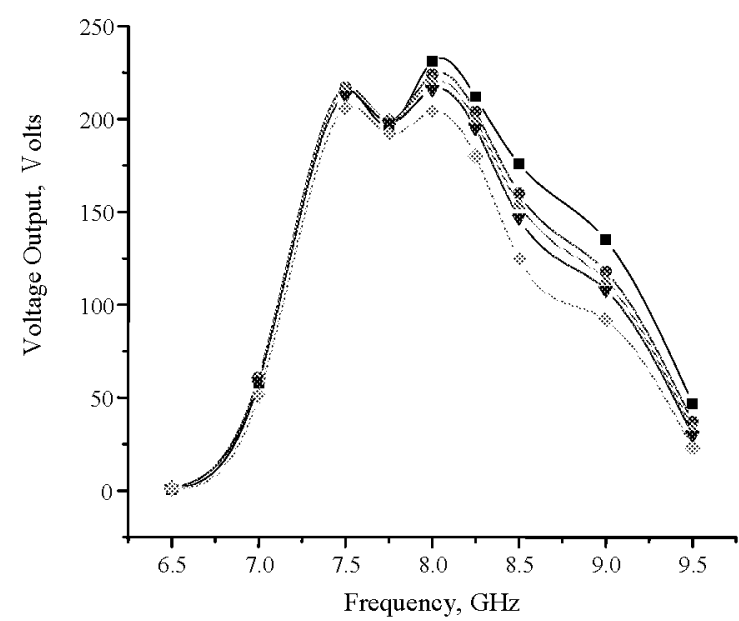

Fig. 7. Frequency dependent voltage outputs from a $6 \times 6$ patch rectenna array measured at various locations of the receiving plane. beam power. This peak voltage output is within the voltage requirement for most piezoelectric actuators.

Fig. 8 shows a THUNDER actuator that was connected to a $6 \times 6$ patch rectenna array for testing. The critical breakdown voltage of a THUNDER actuator similar to the one shown in Fig. 8 was 230 volts. The THUNDER used for the test was rated with a $31 \mathrm{~Hz}$ resonant frequency, 595 volts driving voltage, and a $7.62 \mathrm{~mm}$ maximum displacement. Fig. 9 shows the deflection levels of THUNDER actuator as a function of the voltages fed from the $6 \times 6$ patch rectenna array. A higher voltage output than 230 volts is expected with increase of the beam power. The test of the $6 \times 6$ patch rectenna was limited to the capacity of the 20-watt tunneling-wave tube amplifier (TWTA) used in this study. Currently, a 200-watt TWTA is under installation and should allow for the maximum voltage

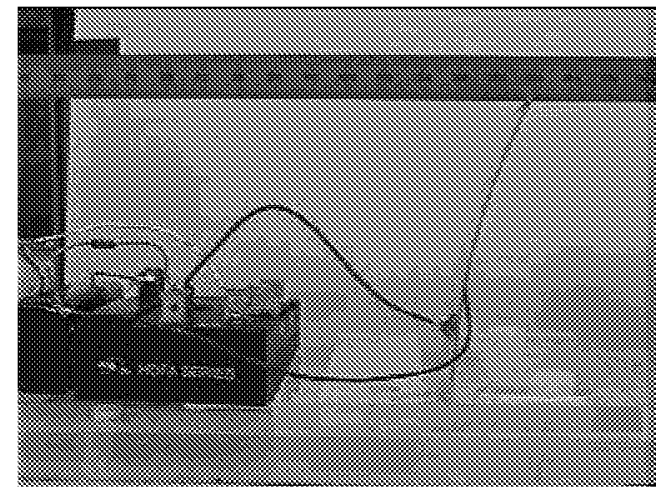

Fig. 8. A THUNDER actuator driven by microwave power output from a $6 \times 6$ patch rectenna. High voltage output is, generally, determined by the number density and breakdown voltage of Schottky barrier diodes in a rectenna array, the beam power, the frequency, and the distance, respectively. The number density of Schottky diodes is determined by how patch rectennas are densely packed in an array at a given frequency. The highest breakdown voltage of commercially available Schottky barrier diodes that are built with GaAs appears to be 9 volts. However, the beam wave dispersion over a distance is an inevitable feature of microwave due to long wavelength and determined by Goubau relation as $\mathrm{D}_{\mathrm{r}}=4 \tau \lambda \mathrm{Z} / \pi \mathrm{D}_{\mathrm{t}}$, where $\tau$ is the constant related to beam transfer efficiency, for example, $\tau=3$ for $100 \%$ transfer efficiency, $\lambda$ the wavelength, $Z$ the distance between transmitting and receiving antennas, and $D_{t}$ the diameter of transmitting

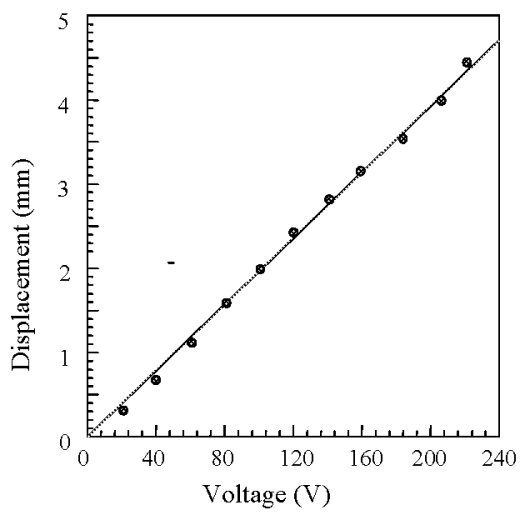

Fig. 9. Deflection of THUNDER actuator 


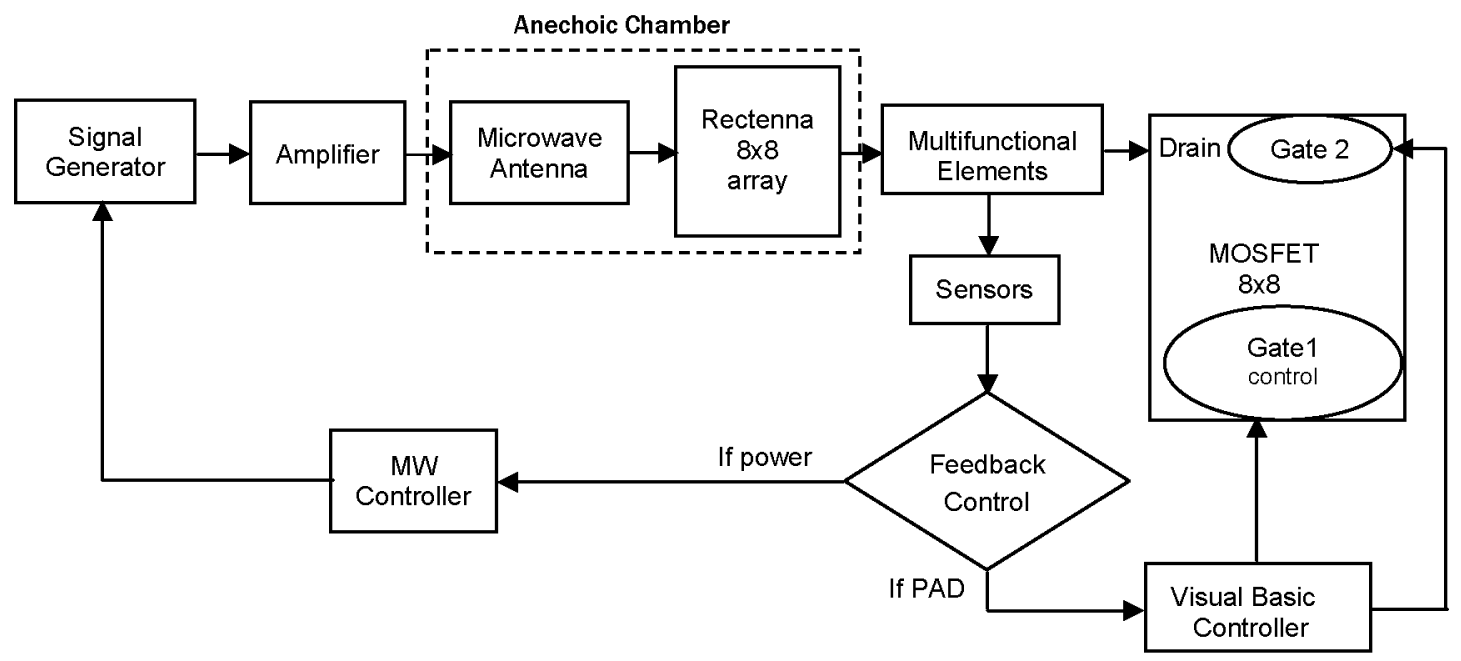

Fig. 10. Block diagram of the system control logic of a networked smart actuators array

antenna. With the $x$-band $(\lambda \approx 0.03$ meter), 0.2 meter of transmitting antenna diameter, and 20 -meters away, the diameter of receiving antenna, $\mathrm{D}_{\mathrm{r}}$ is 11.5 meters. For $\mathrm{K}$-band $(\lambda \approx 0.01$ meter $), \mathrm{D}_{\mathrm{r}}=3.8$ meters. For $\mathrm{W}$-band where $\lambda \approx 0.003$ meter, $\mathrm{D}_{\mathrm{r}}=1.15$ meters. As we see from these simple calculations, the higher the frequency is, the less the dispersion of the wave. Thus, the beam power is concentrated better at a higher frequency. Such a result is also translated into a smaller size of rectenna footprint that will, in turn, increase the number density of rectennas (or Schottky barrier diodes) in an array.

In order to drive actuators or multifunctional elements, an array of rectenna patches are networked in serial mode to provide the required voltage. The networked control logic in a PAD circuitry, in principle, operates to allocate the whole DC power from a rectenna array to a certain region of the actuator array where the surface correction is commanded and then the power is distributed to each individual actuator based on power need. The control logic diagram for the networked multifunctional elements of rectenna array is shown in Fig. 10. The interconnected network circuitry controls logic of all participating rectennas and allocates power to a group of actuators from one location to another location. The networked allocation of power is also regarded as a process of the power amplification corresponding to the power needs for a group of actuators. The logic based on the wireless power feed and PAD concept is composed of a feedback loop of monitoring and determining power flow within the multifunctional element array. The sensors monitoring the performance of the multifunctional element array are integrated and compared to determine power needs for any elements within the array. If the primary power feed must be tailored, the control signal goes to the microwave oscillator to modulate the power or frequency. The eventual scheme for control is to use a microwave signal piggybacked to the main microwave power beam. In Fig. 10, the microwave signal selects which element of the array to be triggered. Once the preprogrammed layout of power distribution is arranged through microwave signals, the respective MOSFETs in an array are gated according to the levels of switching voltages. Gate 2 of MOSFET determines the drain current from a participating actuator element while gate 1 is triggered with a certain voltage level. The challenges ahead for networked smart actuator array development include the development of high voltage hold-off Schottky barrier diodes and dual gate MOSFETs. Hence, the power allocated to the needed area exceeds the threshold power level and energizes the actuators. The PAD circuitry utilizes most of the microwave power at the rectenna's maximum efficiency and tailors the power mode and boosts either the output voltage or output current for the actuator array. The power distribution to a designated group of actuators can be controlled by the external command signals. Fig. 11 shows an illustration for a PAD concept.

Within the group of actuators, the power allocated to the group is distributed to each actuator of the group according to need of power. The power distribution grid will have vertical lines referred to as columns and horizontal lines referred to as rows. These lines are constructed of electrically conducting material. An actuator is placed at the node or intersection of each vertical and horizontal line as shown in Fig. 11. Power 


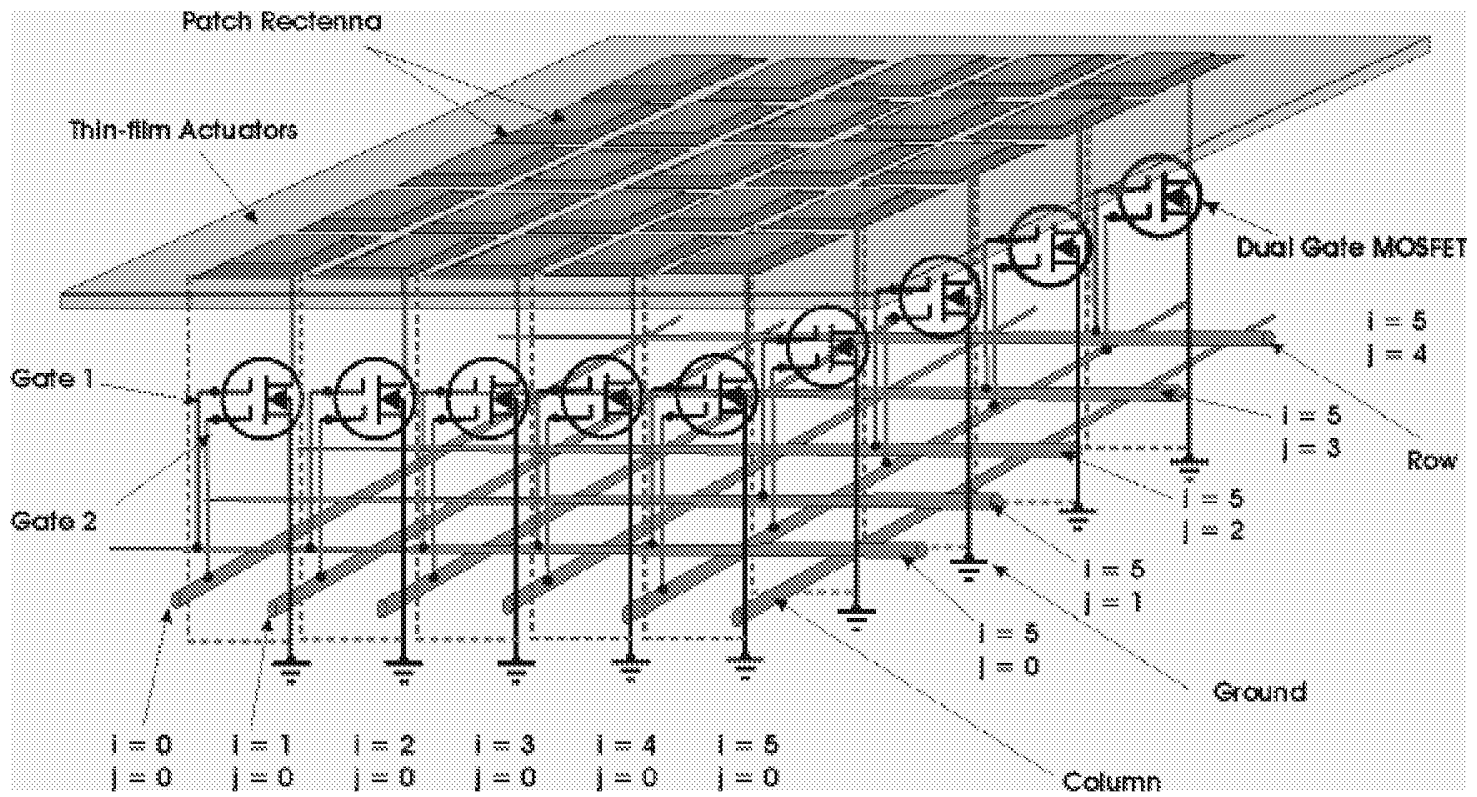

Fig. 11. Schematic of PAD logic circuit

to each actuator can be given from four directions. Such a system insures power being delivered to a given node if some (max. three) of the cross-links should break or be destroyed. If at least one line remains, power will continue to flow to the actuator. Even if all four break, power would still flow and control all the other nodes or actuators in the membrane. The simplest possible component for power distribution would be a variable resistor. An electronic device that can operate as a simple variable resistor is a MOSFET. Enhancement mode power MOSFET's are classified as voltage controlled devices since the drain current $I_{D}$ is controlled by the magnitude of the gate to source voltage, $\mathrm{V}_{\mathrm{GS}}$. When $\mathrm{V}_{\mathrm{GS}}$ is high, typically 10 volts, the drain-to-source resistance falls to a very low value (typically less than $1 \Omega$ ). $I_{D}$ is also limited by the impedance of the load and the magnitude of the drain supply voltage. Applying a low voltage across the gateto-source, simply 0 volts, the drain-to-source resistance increases (typically several $\mathrm{M} \Omega$ ) and turns the MOSFET off. Thus one MOSFET can function as the control element to one piezoelectric displacement actuator.

To control or address each MOSFET, the control logic used to refresh DRAM in a personal computer can be used. The memory logic voltage, 0 or 5 volts, is stored in a capacitor inside the memory. This capacitor will discharge if not refreshed periodically. Rows and columns are energized systematically in a swept fashion to recharge the memory capacitors. In a similar fashion, individual nodes in the distribution power grid can be addressed if the corresponding row and column are driven high. This solution implies that we have a MOSFET with two identical gate inputs capable of turning off the MOSFET independently. In a true AND gate fashion, the MOSFET will only conduct when both gate inputs (row and column) are high.

Most discrete MOSFET's used today have one gate drive. In early days, dual gate MOSFET's were used in RF mixers and automatic gain controlled IF (intermediary frequency) stage electronics. With the fabrication of ever increasing complex integrated circuits, the low demand for dual gate FET's has foreed many manufacturers to stop making them. We were only able to obtain discontinued Motorola dual-gate depletion mode MOSFETs, the MFE201. The depletion mode operation forced the control circuitry to use negative gate drive voltages in order to turn the MOSFET off.

\section{CONCLUSION}

From the experimental results, it was clear that that the rectenna array generates sufficient power to drive multifunctional elements. The wireless power transmission by microwave eliminates the need for the hard-wiring of multifunctional elements on the adaptive surfaces of a large space deployable reflectors 
or inflatable antennas. Hence, it could dramatically reduce the total cost and weight of the multifunctional systems and find applications in many challenging space missions. The conceptual design of PAD presented is a reasonable solution to the complexity of wiring many actuators in a smart material. The design is simple enough to imbed into a thin film microcircuit layer during the fabrication of the smart material. For high power applications, high voltage hold-off Schottky barrier diodes and MOSFETs are essential for driving actuators with a large displacement requirement, such as aircraft morphing actuators which require $>200$ volts.

\section{ACKNOWLEDGMENTS}

This work was performed partially under the NASA grant (NCC1-280) to Norfolk State University.

\section{REFERENCES}

1. Sang H. Choi, Lake, M., and Moore, C., "Microwave-driven Smart Material Actuators." Patent Pending, NASA Case No. LAR 15754-1, Feb 24, 1998.

2. Sang H. Choi, S. Chu, M. Kwak and A. D. Cutler, "A Study on a Microwave-driven Smart Material Actuator," SPIE Symposium on Smart Structures and Materials, 1999.

3. I. V. Galaev, M. N. Gupta, and B. Mattiasson, "Use Smart Polymers for Biosepartions," CHEMTECH, No. 12, pp. 19-25, 1996.

4. T. K. Wu, "Piezoelectrically adjustable array for large reflector antenna surface distortion compensation," Microwave and Optical Technology Letters, Vol. 14, No. 4, pp. 221-224, 1997.

5. J. Huang, T. K. Wu, and S. W. Lee, "Tri-Band FSS with Circular Ring Elements," IEEE Trans. Antennas Propagat., vol. AP-42, No. 2, pp. 166-175, 1994.

6. T. K. Wu, "Multiband FSS," in T. K. Wu (Ed.), Frequency Selective and Grid Array, John and Wiley \& Sons, New York, 1995.

7. W. Schneider, J. Moore, T. Blankney, D. Smith, and J. Vacchione, "An Ultra-Lightweight High Gain Spacecraft Antenna," IEEE Antennas Propagat. Int. Symp., Seattle, WA, June, pp. 886-889, 1994.

8. T. H. Lee, R. C. Rudduck, T. K. Wu, and C. Chandler, "Structure Scattering Analysis for SeaWinds Scatterometer Reflector Antenna Using Extended Aperture Integration and GTD," IEEE Antennas propagat. Int. Symp., pp. 890-893, Seattle, WA, June, 1994.
9. W. C. Brown, et al., U.S. Patent 3434 678, Mar. 25,1969 .

10. W. C. Brown, "Experiments involving a microwave beam to power and position a helicopter," IEEE Trans. Aerosp. Electron. Syst., Vol. AES-5, No. 5, pp. 692-702, 1969.

11. W. C. Brown, "Solar Power Satellite Program Rev. DOE/NASA Satellite Power System Concept Develop. Evalation Program," Fianl Proc. Conf. $800491,1980$.

12. J. Schlesak, A. Alden and T. Ohno, " A microwave powered high altitude platform," IEEE MTT-S Int. Microwave Symp. Dig., pp. 283-286 1988.

13. W. C. Brown, "Design study for a ground microwave power transmission system for use with a high-altitude powered platform," NASA Final Contractor Report 168344, Raytheon Rpt. PT-6052, 1983.

14. NASA JPL, "Patch Rectenna for Converting Microwave to DC Power," NASA Tech Briefs, Vol. 21, January, p. 40, 1997.

15. K. Lubitz and H. Hellebrand, "Properties of PZT Multilayer Actuators," IEEE 7th Inter. Symp. Applications of Ferroelectrics, pp. 509-512, 1991.

16. W. C. Brown, "Rectenna Technology Program: Ultra Light $2.45 \mathrm{GHz}$ Rectenna and $20 \mathrm{GHz}$ Rectenna," Raytheon Company PT-6902, NASA Contract Report, CR179558, March 11, 1987. 